\title{
Multi-factor jump-diffusion models of electricity prices*
}

\author{
Thilo MEYER-BRANDIS \\ Center of Mathematics for Applications \\ University of Oslo \\ P.O. Box 1053, Blindern, Norway \\ e-mail: meyerbr@math.uio.no
}

\author{
Peter TANKOV \\ Laboratoire de Probabilités et \\ Modèles Aléatoires \\ Université Paris-Diderot (Paris 7) \\ e-mail: tankov@math.jussieu.fr
}

\begin{abstract}
The recent deregulation of electricity markets has led to the creation of energy exchanges, where the electricity is traded like any other commodity. In this paper, we study the most salient statistical features of electricity prices with a particular attention to the European energy exchanges. These features can be adequately reproduced by the sum-OU model: a model representing the price as a sum of Lévy-driven OrnsteinUhlenbeck (OU) processes. We present a new method for filtering out the different OU components and develop a statistical procedure for estimating the sum-OU model from data.
\end{abstract}

Key Words: electricity prices, multi-factor models, Lévy-Driven OrnsteinUhlenbeck type processes, statistical estimation, nonlinear filtering

\section{Introduction}

Similarly to how the end of the Bretton Woods system in 1973 triggered the appearance of currency exchanges, the recent deregulation of electricity markets has led to the creation, in Europe, United States and other countries of a network of energy exchanges, where the electricity is quoted almost as any other commodity. The power prices, which were fixed by governments before competition was introduced, are now determined via an equilibrium of supply and demand, and present a much higher volatility than equity prices. A precise statistical model of electricity spot price behavior is therefore necessary for energy risk management, pricing of electricity-related options and evaluation of production assets.

This paper focuses on statistical modeling of spot electricity prices with a particular attention to the (more or less recently established) European energy

*This research project was supported by the Europlace Institute of Finance 
exchanges. The electricity prices are usually quoted on hourly basis, however, they cannot be seen as one long hourly series, because in most markets, the delivery prices for all 24 hours of a given day are determined by the system operator simultaneously on the previous day (day-ahead prices). Depending on the market, this may happen between 11AM and 1PM of the previous day. After this, the day-ahead market closes and only small changes can be made to delivery contracts using the adjustment market in case of emergency such as plant outage. There is thus no causality relationship between different hourly prices on the same day, and the hourly electricity data should rather be seen as 24 dependent daily series than one long hourly series. Given the structure of the day-ahead electricity market, a possible model for hourly prices would be

$$
X_{t}^{h}=Y_{t} f(t, h)+\varepsilon_{t}^{h},
$$

where

- $X_{t}^{h}, h \in\{1, \ldots, 24\}$ is the price for day $t$ and hour $h$;

- $Y_{t}$ is the common factor (average daily price);

- $f(t, h)$ is a slowly varying daily pattern;

- $\varepsilon_{t}^{h}$ is a white noise.

A principal component analysis on the Powernext hourly data shows that the first component corresponding to a constant daily pattern $f(t, h)=f(h)$ explains $70 \%$ of variance. Therefore, most of the variability of electricity prices, as well as all interesting statistical features like mean reversion are contained in the average daily price series, and the daily pattern is mostly related to seasonality. In this paper, we therefore concentrate on modeling the daily electricity price series.

The rest of this paper is structured as follows. In Section 2 we elucidate the qualitative features of electricity prices common for different data sets that we study. Section 3 discusses different models for electricity spot prices found in the literature and how well they describe the stylized features of the data. At the end of this section, we introduce our model of choice: the sum-OU model, where the spot price is described as sum of several non-Gaussian Ornstein-Uhlenbeck processes. This type of model was introduced in [3], and it both captures well the stylized facts of electricity spot prices and is analytically tractable for derivatives pricing and risk management. However, the important question of how to estimate and implement the model has not yet been addressed in the literature. The rest of this paper is therefore dedicated to the estimation of this model from data: Section 4 shows how different components can be separated, and Section 5 presents a detailed case study where the sum-OU model is estimated completely from the EEX spot price series.

While the main objective of this work is to provide an estimation procedure for the multi factor non-Gaussian OU model, the original contribution in this paper is threefold: 
- We discuss the stylized features of electricity prices some of which are being reported here for the first time. In addition, we focus on quotes from European energy exchanges which are much less studied than the American ones (except maybe the Nord pool market) and present qualitatively different characteristics.

- We propose a novel method for estimating the sum-OU model from price data, based on recent findings in nonparametric statistics.

- We give a complete data-driven specification of the model and present a detailed case study where all parameters of this specification are estimated from EEX time series.

The principal application of our model lays in simulating market evolution scenarios for computing risk measures, testing hedging strategies and evaluating investment policies.

\section{$2 \quad$ Stylized features of electricity prices}

This section discusses qualitative features of electricity prices common for different data sets that we study.

Description of data sets Our study uses the following six sets of daily data (weekends removed), provided by Datastream. The last date in all series is $21 / 11 / 2006$.

- Dow-Jones California-Oregon border firm on-peak electricity price index (COB) starting 19/05/1997.

- Dow-Jones Mead/Marketplace firm on-peak electricity price index (MEAD) starting 08/08/2000.

- European Power Exchange Phelix Base electricity price index (EEX) starting 16/06/2000.

- Amsterdam Power Exchange daily average (APX) starting 26/05/1999.

- United Kingdom Power Exchange daily average (UKPX) starting 27/03/2001.

- Nord Pool system price daily average (NP) starting 1/1/1994.

For some illustrations we also use the Powernext (French energy exchange) daily data downloaded from Powernext website. 


\begin{tabular}{r|cccc} 
Series & Intraweek exp. ret. & FM exp. ret. & Intraweek var. & FM var. \\
\hline & & & & \\
COB & $-0.015(0.003)$ & $0.061(0.007)$ & $0.019(0.002)$ & $0.028(0.006)$ \\
MEAD & $-0.011(0.003)$ & $0.042(0.007)$ & $0.014(0.001)$ & $0.018(0.003)$ \\
EEX & $-0.006(0.006)$ & $0.027(0.014)$ & $0.050(0.006)$ & $0.062(0.010)$ \\
APX & $-0.016(0.007)$ & $0.068(0.022)$ & $0.083(0.008)$ & $0.191(0.035)$ \\
UKPX & $-0.013(0.005)$ & $0.054(0.014)$ & $0.025(0.002)$ & $0.055(0.013)$ \\
NP & $-0.005(0.001)$ & $0.019(0.004)$ & $0.006(0.0006)$ & $0.012(0.002)$
\end{tabular}

Table 1: Expectations and variances of intraweek and Friday to Monday returns. The standard deviations of the estimators, computed by bootstrap, are given in brackets.

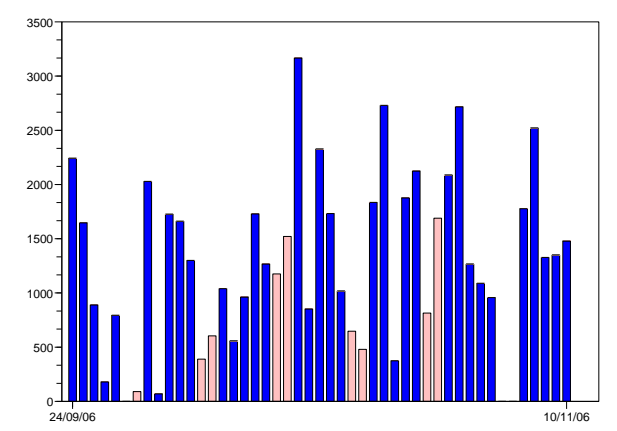

Figure 1: Daily trade volume, in MWh, on the EEX market, from 24/09/2006 to $10 / 11 / 2006$. Bright-colored bars correspond to weekends. Data downloaded from EEX website at www.eex.de.

Weekend effect As shown in Table 1, the distribution of electricity index returns from Friday to the following Monday is significantly different from the distribution of intraweek daily returns for all six series under study. First of all, the variances of weekend returns are 1.2 to 2 times larger than those of intraweek returns. This is explained by the fact that unlike stocks, electricity is traded on the weekends, although in much smaller volumes (see Fig. 1). Secondly, for all six series under study, the expected Friday to Monday return is reliably positive whereas the intraweek returns are negative or indistinguishable from zero. This is behavior is opposite to what has been observed for equity returns $[8,16]$. While weekends introduce a lot of seasonality into the price series they don't account for interesting statistical features (e.g., no spikes during weekends). In order to make deseasoning easier and to concentrate on statistical aspects, we consider time series without weekends. 


\begin{tabular}{l|cc} 
Series & Affine trend $R^{2}$ & Seasonal trend $R^{2}$ \\
\hline COB & 0.0990 & 0.1031 \\
MEAD & 0.0274 & 0.0385 \\
EEX & 0.5160 & 0.5373 \\
APX & 0.1378 & 0.1715 \\
UKPX & 0.5615 & 0.5900 \\
NP & 0.2504 & 0.3106
\end{tabular}

Table 2: $R^{2}$ (proportion of initial variance explained by the trend function) with seasonality and without it (in this case, the trend function is affine).
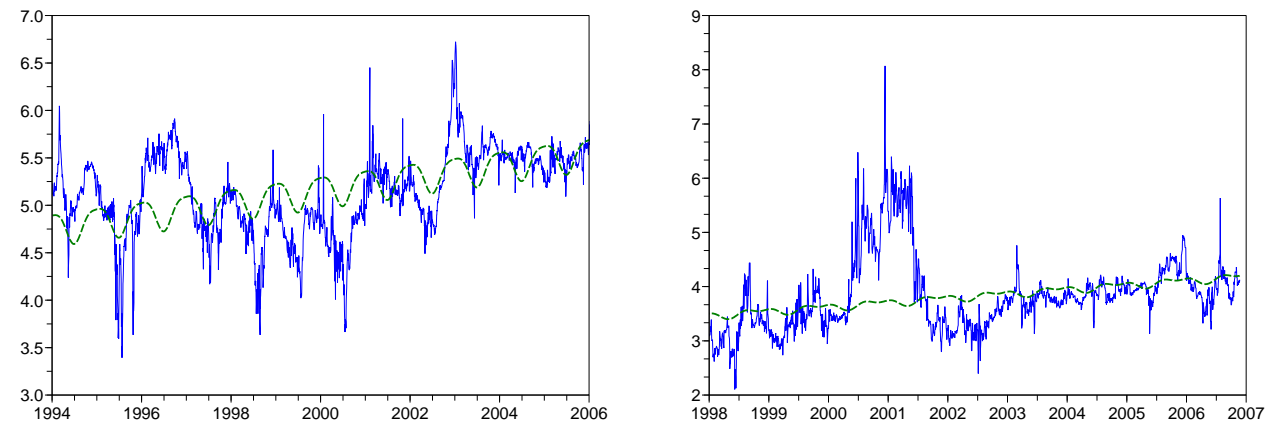

Figure 2: Left: Nord Pool series (strongest seasonal effect). Right: COB series (weakest seasonality).

Yearly seasonality Many studies (see for instance [10]) have established the presence of annual seasonality in electricity spot prices. Following [10], we remove the seasonality by representing it as a linear combination of sinusoids with periods 12 and 6 months. We estimate the seasonality by fitting a 6 parameter trend function

$$
f(t)=a+b t+c_{1} \sin (2 \pi t)+c_{2} \cos (2 \pi t)+d_{1} \sin (4 \pi t)+d_{2} \cos (4 \pi t)
$$

to the log-price series by ordinary least squares. A linear trend and both harmonics are statistically significant at a $1 \%$ level in all 6 six series under study. However as seen from the Table 2, the seasonality contributes very little to the overall variability of the series. The proportion of variance explained by seasonality is highest for the Nord Pool series (whose huge share of hydro energy highly depends on weather cycles) and lowest for the COB series. These two series are represented in Figure 2. From now on, we concentrate on the deseasonalized series, that is, on the residuals of the least squares regression.

Stationarity and mean reversion Contrary to stock prices, the electricity prices tend to exhibit stationary behavior. For all six series under study, the 


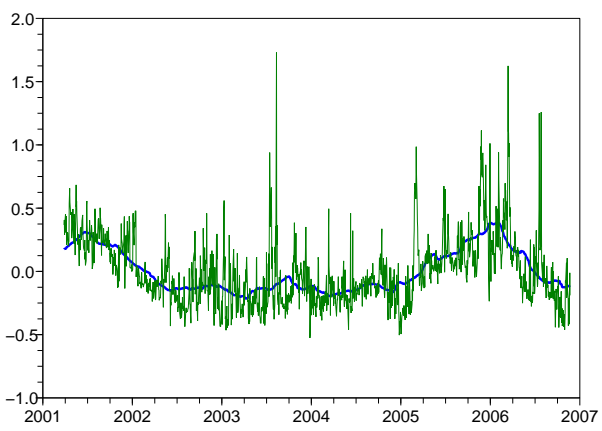

Figure 3: UKPX time series (after the seasonality has been removed) and its 6 -month moving average.

\begin{tabular}{l|cccccc} 
Series & APX & COB & EEX & MEAD & NP & UKPX \\
\hline $\begin{array}{l}\text { Standard DF test on desea- } \\
\text { sonalized series }\end{array}$ & 485 & 59.5 & 505 & 43.4 & 71.9 & 304 \\
$\begin{array}{l}\text { DF test with linear trend on } \\
\text { raw series }\end{array}$ & 466 & 59.4 & 483 & 43.2 & 66.4 & 284 \\
& & & & & &
\end{tabular}

Table 3: Dickey-Fuller unit root test statistics for the six price series under study. For all six series the non-stationarity hypothesis is rejected at $1 \%$ confidence level. For the standard test, the non-stationarity hypothesis is rejected at $1 \%$ level if the statistics is greater than 13.8. For the test with linear trend, it is rejected if the statistics is greater than 29.5 .

unit root hypothesis was confidently rejected by the Dickey-Fuller test, both on the deseasonalized data and on the non-deseasonalized one (in the latter case, a variant of the Dickey-Fuller test with a linear trend was used). The test statistics are reproduced in Table 3.

The electricity prices, similarly to other commodities, are therefore mean reverting to a trend which may exhibit slow stochastic variations as in Figure 3

Spikes A fundamental property of electricity spot prices, already observed by many authors, and present in all 6 time series studied in this paper, is the presence of spikes, that is, rapid upward price moves followed by a quick return to about the same level. Typical spike profile for Nord Pool price series is shown in Figure 4. During the peak period, the price process has different properties. In particular, the rate of mean reversion is much higher than during normal evolution. The presence of spikes is a fundamental feature of electricity prices due to the non-storable nature of this commodity and any relevant spot price model must take this feature into account.

The presence of long-term stochastic variation in the mean level of electricity 


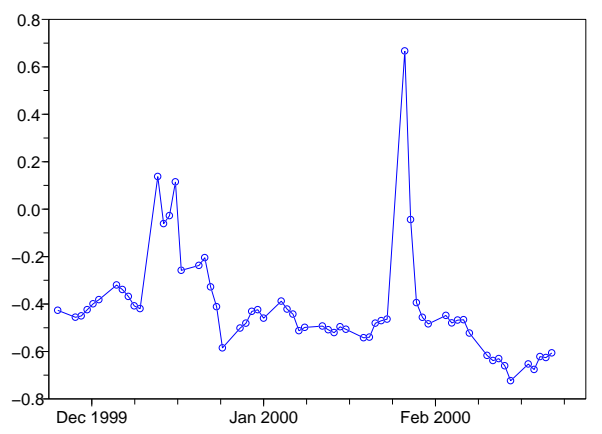

Figure 4: Spikes in the Nord Pool price series (after logarithmic transformation and seasonal trend removal).

$\begin{array}{cccccc}\text { APX } & \text { COB } & \text { EEX } & \text { MEAD } & \text { NP } & \text { UKPX } \\ 513 \% & 231 \% & 363 \% & 197 \% & 133 \% & 282 \%\end{array}$

Table 4: Annualized volatilities of electricity prices.

prices makes it difficult to establish a range of prices, for which the price process is in peak mode: what is considered as peak level now, may become normal in 3 years. The rate of mean reversion is therefore determined not only by the current price level but also by the previous evolution of the price, which suggests that the behavior of electricity spot prices may be non-markovian.

As observed in [10], the intensity of spikes can exhibit yearly seasonal patterns. We will come back to this issue in Section 4 devoted to spike detection.

Multiscale autocorrelation The examination of autocorrelation structure of the six electricity price series (Figure 5) reveals the presence of two very distinct types of behavior: the autocorrelations of COB, MEAD and NP series decay very slowly, while the autocorrelation function of APX, EEX, and UKPX series present a two-scale behavior with a quickly decaying component and a slow one. This striking difference may be due to differences in the organization of electricity markets [9]: the APX, EEX and UKPX markets are organized as power exchanges where the reported price is simply the price of the last transaction. On the other hand, the price index we use for the Nord Pool market is the so-called system price resulting from the bids of all market participants, and the $\mathrm{COB}$ and MEAD price series are indices compiled by Platts as average of all transactions reported by market participants [15]. This suggests that APX, EEX and UKPX prices will have a higher volatility and faster mean reversion than COB, MEAD and NP, which is exactly what is observed in Figure 5 and Table 4. 

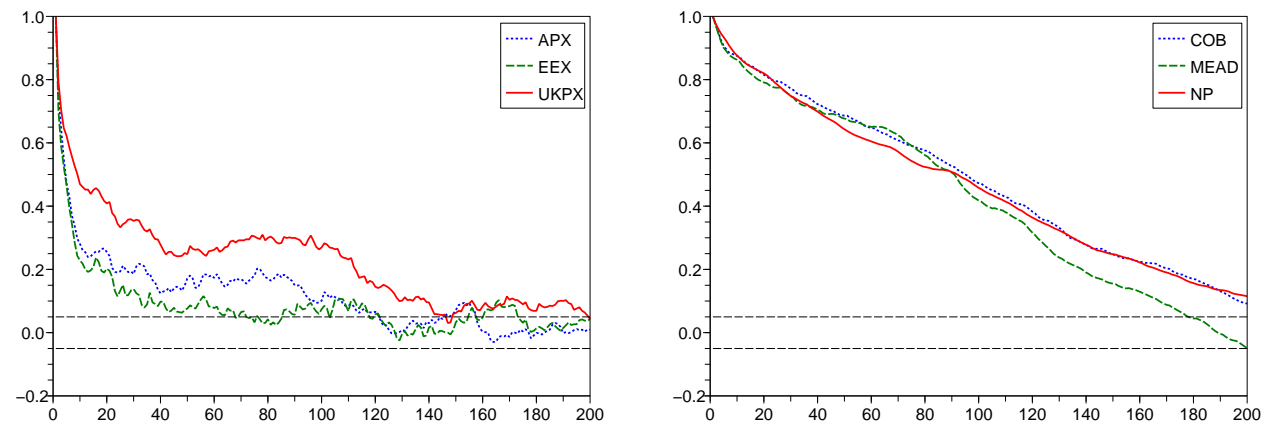

Figure 5: Autocorrelation functions of electricity price series.

$\begin{array}{cccc} & \text { APX } & \text { EEX } & \text { UKPX } \\ w_{1} & 0.300 & 0.161 & 0.468 \\ \lambda_{1} & 81.5 & 94.3 & 112.6 \\ w_{2} & 0.688 & 0.761 & 0.483 \\ \lambda_{2} & 2.9 & 4.3 & 3.7\end{array}$

Table 5: Fitting the autocorrelation function with a sum of two exponentials.

Let us now focus on the study of the multiscale autocorrelation structure in APX, EEX and UKPX time series. The observed structure is described quite precisely with a sum of two exponentials:

$$
\rho(h)=w_{1} e^{-h / \lambda_{1}}+w_{2} e^{-h / \lambda_{2}} .
$$

Figure 6 and Table 5 show the quality of the fit and the fitted parameters. This type of correlation structure arises in a model where the price is a sum of two independent mean-reverting components with two different rates of mean reversion. We conjecture that in this context, the fast rate of mean reversion, corresponding to correlation length between 3 and 5 days corresponds to the "spike mode" while the second slower rate of mean reversion, with correlation length of about 100 days, corresponds to normal price evolution. This idea will be further developed in the subsequent sections.

Non-gaussian distribution of returns The descriptive statistics of return distributions (skewness and kurtosis) shown in Table 6 highlight the non-gaussian character of electricity spot returns. This is explained by the presence of spikes in the data since these low-probability large-amplitude moves clearly cannot arise in a Gaussian framework. First of all, all six distributions are strongly leptokurtic, with excess kurtosis values ranging from 9 to 29. For comparison, the excess kurtosis of daily returns of the S\&P 500 index is about 3. Secondly all six series are slightly positively skewed. This is related to the fact that the 

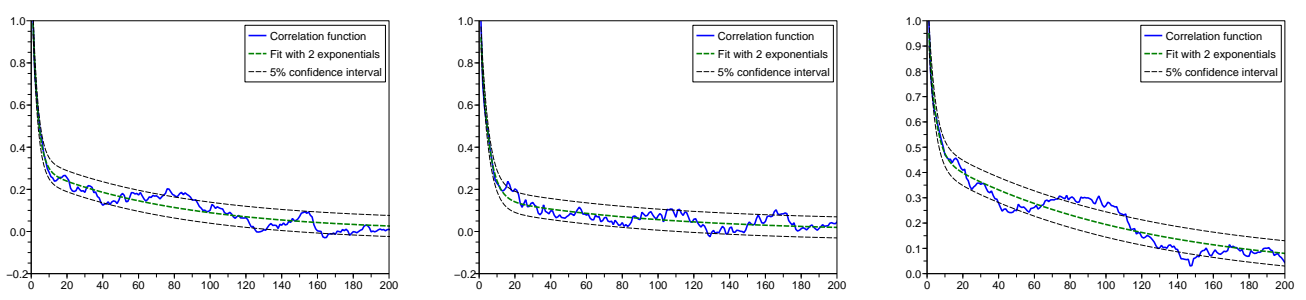

Figure 6: Fitting the autocorrelation function with a sum of two exponentials. From left to right: APX, EEX, UKPX.

downward price move at the end of a spike is not quite as fast as the upward move in the beginning and can last several days.

Figure 7 plotting the logarithm of the empirical distribution function for the right and left tail of EEX return distribution shows that these tails are fatter than exponential. In case of exponential tail decay, the empirical distribution function

$$
F_{n}(x)=\frac{1}{n} \sum_{i=1}^{n} 1_{X_{i} \leq x}
$$

satisfies

$$
F_{n}(x) \sim e^{-\lambda_{1}|x|} \text { as } x \rightarrow-\infty \text { and } 1-F_{n}(x) \sim e^{-\lambda_{2} x} \text { as } x \rightarrow \infty .
$$

Therefore $\log F_{n}(x)$ must behave like a linear function when $x \rightarrow-\infty$ and similarly for the right tail. However, this is not what is observed in Figure 7: the graph is convex and we conclude that both tails are fatter than exponential. The same conclusion holds for all 6 series that we study.

To detect the possible power-law behavior in the tails of return distribution we use the Hill estimator. To fix the ideas, let us concentrate on the upper tail of the distribution and suppose that the distribution function satisfies

$$
1-F(x) \sim x^{-\alpha} L(x), \quad x \rightarrow \infty, \alpha>0,
$$

where $L$ is a function satisfying $\lim _{t \rightarrow \infty} L(t x) / L(t)=1$ for all $x$ (slowly varying function). Then the extreme value index $\gamma=\alpha^{-1}$ can be estimated with the Hill estimator defined by

$$
H_{k, n}:=\frac{1}{k} \sum_{i=1}^{k} \log \frac{X_{(i)}}{X_{(k+1)}},
$$

where $\left(X_{(i)}\right)$ are order statistics. This estimator is consistent under a broad set of assumptions (see [4] for a review). In practice, if $H_{k, n}$ remains stable over a large range of $k$, this is an indication of power-like tail behavior.

Looking at the data (see Figure 8), we see that for all six series under study, in the range between $k=30$ and $k=150$, the Hill plot stabilizes around the 


\begin{tabular}{l|cccccc} 
Series & APX & COB & EEX & MEAD & NP & UKPX \\
\hline Skewness & 0.11 & 0.163 & 0.641 & 0.025 & 0.431 & 0.801 \\
Excess kurtosis & 14.8 & 15.4 & 12.7 & 9.5 & 29.0 & 15.0
\end{tabular}

Table 6: Descriptive statistics of electricity return distributions.
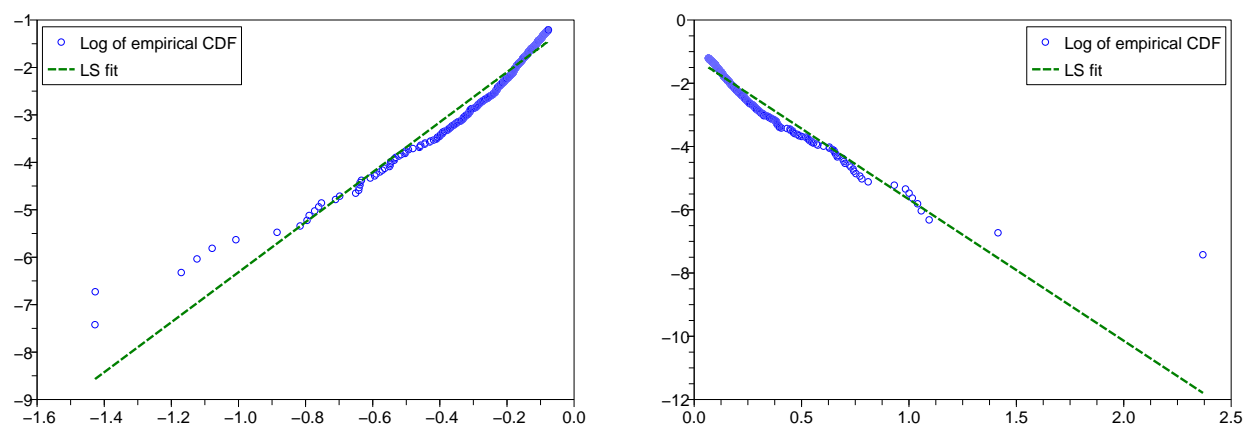

Figure 7: Tails of the empirical distribution function of EEX returns. The left graph shows $\log F_{n}(x)$ and the right graph $\log \left(1-F_{n}(x)\right)$

value between 0.3 and 0.5 (for both tails). This suggests that the tail behavior of electricity prices may be well described by a power law with decay parameter $2<\alpha<3$. Electricity returns have thus finite second moment which justifies the use of autocorrelation functions above in this section.

\section{Literature review}

In this section we will give a short overview of the main types of continuous time reduced form spot price models from the existing literature. We mention that there also exist a variety of econometric time series models (see e.g. [7, 12, 13, 14] and references therein). However, we don't go further into this line of research here.

Structural models In structural or equilibrium models $[1,11]$, one tries to mimic the price formation in electricity market as a balance of supply and demand. The (very inelastic) demand for electricity is described by a stochastic process:

$$
\begin{aligned}
& D_{t}=\overline{D_{t}}+X_{t}, \\
& d X_{t}=\left(\mu-\lambda X_{t}\right) d t+\sigma d W_{t},
\end{aligned}
$$

where $\overline{D_{t}}$ describes the seasonal component and $X_{t}$ corresponds to the stationary stochastic part. The price is obtained by matching the demand level with 

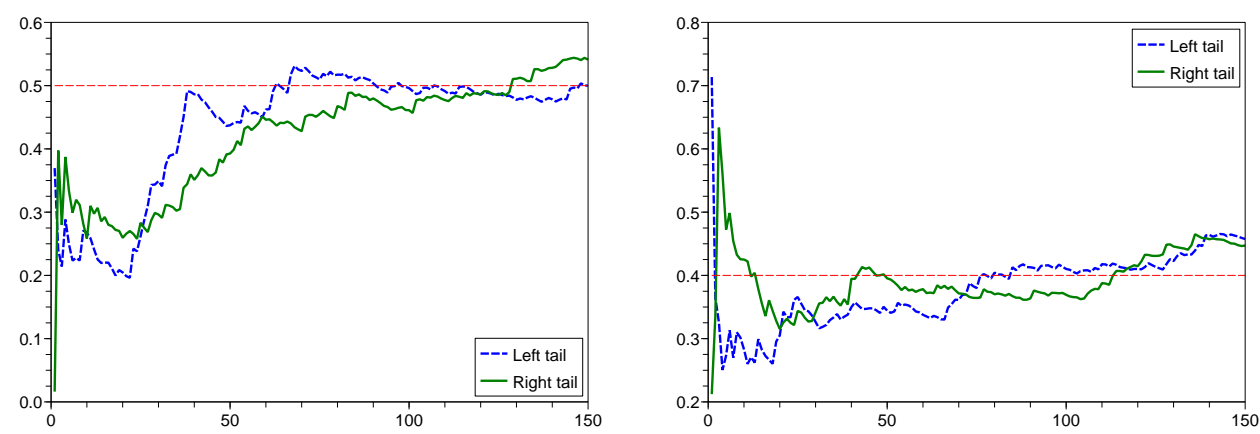

Figure 8: Hill plots for MEAD (left) and UKPX (right) series.

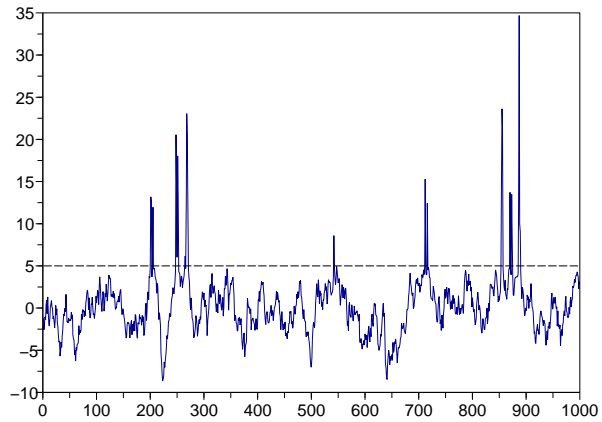

Figure 9: Spot price trajectory in the structural model of Kanamura and Ohashi $[11]$.

a deterministic supply function which must be nonlinear to account for the presence of price spikes. Barlow [1] proposes

$$
P_{t}=\left(\frac{a_{0}-D_{t}}{b_{0}}\right)^{1 / \alpha}
$$

for some $\alpha>0$ while Kanamura and Ohashi [11] suggest a "hockey stick" profile

$$
P_{t}=\left(a_{1}+b_{1} D_{t}\right) 1_{D_{t} \leq D_{0}}+\left(a_{1}+b_{1} D_{t}\right) 1_{D_{t}>D_{0}} .
$$

A typical spot price trajectory in the latter model is shown in Figure 9.

While structural models reproduce price spikes on a qualitative level, the assumption of deterministic supply function is probably too restrictive. It implies in particular that spikes can only be caused by surges in demand, while in electricity markets spikes can also be due to sudden changes in supply, such 
as plant outages. In addition, prices in such models do not have the multiscale autocorrelation property observed in the data, and, with a deterministic supply function, do not allow for a stochastic base level as in Figure 3.

Markov models To make estimation easier, Geman and Roncoroni [10] model the electricity log-price as a one-factor Markov jump diffusion.

$$
d P_{t}=\theta\left(\mu_{t}-P_{t}\right) d t+\sigma d W_{t}+h(t) d J_{t}
$$

The spikes are introduced by making the jump direction and intensity leveldependent: if the price is high, the jump intensity is high and downward jumps are more likely, whereas if the price is low, jumps are rare and upward-directed. This approach produces realistic trajectories and reproduces the seasonal intensity patterns observed in American price series, but once again, the process reverts to a deterministic mean level rather than the stochastic pre-spike value.

Regime-switching models In the one-factor Markov specification of Geman and Roncoroni [10], the 'spike regime' is distinguished from the 'base regime' by a deterministic threshold on the price process: if the price is higher than a given value, the process is in the 'spike regime' otherwise it is in the 'base regime'. This threshold value may be difficult to calibrate and it is not very realistic to suppose that it is determined in advance. The regime-switching models of Deng [5] and Weron [18] alleviate this problem by introducing a two state unobservable markov chain which determines the transition from "base regime" to "spike regime" with greater volatility and faster mean reversion:

$$
\begin{array}{ll}
d P_{t}=\theta^{1}\left(\mu_{t}-P_{t}\right)+\sigma^{1} d W_{t} & \text { (base regime) } \\
d P_{t}=\theta^{2}\left(\mu_{t}-P_{t}\right)+\sigma^{2} d W_{t} & \text { (spike regime). }
\end{array}
$$

This nonlinear model is more difficult to estimate than a one-factor Markov specification (filtering must be used) and once again, the stochastic base level is not taken into account since in the spike regime, the process quickly reverts to the seasonal mean and not to the pre-spike base level.

Multifactor models To allow mean reversion to a stochastic base level, several authors have suggested to model the price as sum of several factors. In the simplest case of a two-factor model, the first factor corresponds to the base signal with a slow mean reversion, and the second factor represents the spikes and has a high rate of mean reversion. The first models of this type (see [2] and references therein) reprensented the electricity price (or log-price) as sum of Gaussian Ornstein-Uhlenbeck processes. However, while the first factor can in principle be a Gaussian process, the second factor is close to zero most of the time (when there is no spike) and takes very high values during a spike. This behavior is hard to describe with a Gaussian process, and several authors $[3,6,17]$ have therefore explored a jump-diffusion specification. 
In Benth, Kallsen and Meyer-Brandis [3], the deseasonalized spot price is a sum of independent Lévy-driven Ornstein-Uhlenbeck components:

$$
\begin{aligned}
& X(t)=\sum_{i=1}^{n} Y_{i}(t) \\
& d Y_{i}(t)=-\lambda_{i}^{-1} Y_{i}(t) d t+d L_{i}(t), \quad Y_{i}(0)=y_{i},
\end{aligned}
$$

and processes $L_{i}(t)$ are independent, possibly time inhomogeneous Lévy processes with $E\left[L_{i}^{2}(1)\right]<\infty$.

In this model, the autocorrelation function $\rho_{t}(h)$ defined as the correlation between $X(t)$ and $X(t-h)$ satisfies

$$
\rho_{t}(h):=\frac{\sum_{i=1}^{n} w_{i}(t) e^{-h / \lambda_{i}}}{\sum_{i=1}^{n} w_{i}(t)}
$$

In the stationary case (i.e. the underlying processes are time homogeneous Lévy processes), we have simply

$$
\rho(h):=\frac{\sum_{i=1}^{n} w_{i} e^{-h / \lambda_{i}}}{\sum_{i=1}^{n} w_{i}} \quad \text { with } \quad w_{i}=\lambda_{i} \operatorname{Var} L_{i}(1) .
$$

This modeling approach thus allows to explain the multiscale autocorrelation phenomenon observed in European spot price series in Section 2

Figure 10 shows a typical price trajectory in this model, with two factors, one of which is driven by a Brownian motion and another one by a compound Poisson process (with Gaussian jump sizes).

This model, coupled with a good description of price seasonality, provides a precise characterization of electricity spot price behavior, especially well suited to European energy markets. In addition, due to its arithmetic structure, it is analytically tractable when it comes to futures and other derivatives pricing. In the remaining part of this paper we will discuss the question of fitting this model to data.

\section{Detecting and filtering the spikes}

For the remaining parts of the paper we will deal with the question of how to estimate a multi-factor model as presented in (2)-(3). In particular, we will focus on the following two factor specification for the deseasonalized spot price (eventually log-spot price) $X(t)$ :

$$
\begin{aligned}
& X(t)=Y_{1}(t)+Y_{2}(t) \\
& d Y_{i}(t)=-\lambda_{i}^{-1} Y_{i}(t) d t+d L_{i}(t), \quad i=1,2 .
\end{aligned}
$$

Here $Y_{2}(t)$, the Ornstein-Uhlenbeck component responsible for spikes, is driven by a Lévy processes $L_{2}(t)$ with possibly time varying jump intensity. The 


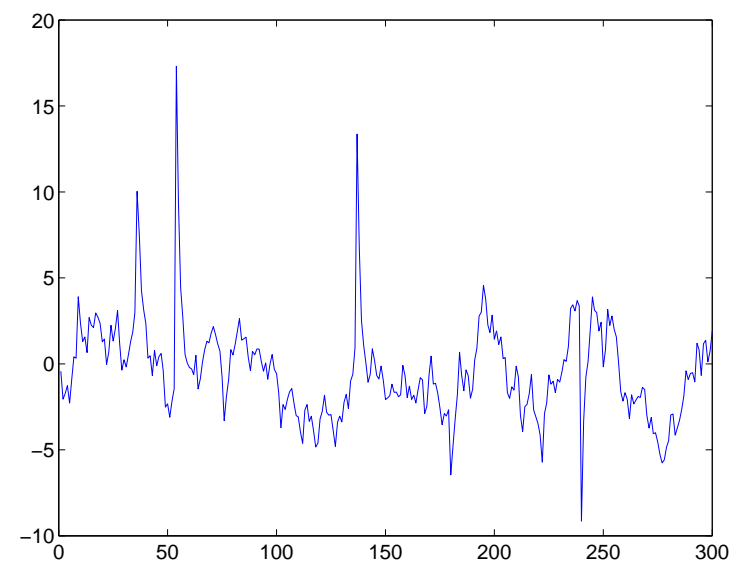

Figure 10: Sample spot price trajectory in a two-factor non-Gaussian OrnsteinUhlenbeck model.

Ornstein-Uhlenbeck component $Y_{1}(t)$, responsible for the remaining price variations, is assumed to be Gaussian, i.e. driven by a Brownian motion $L_{1}(t)$. We recall that the Ornstein-Uhlenbeck components (5) are explicitly given by

$$
Y_{i}(t)=e^{-(t-s) / \lambda_{i}} Y_{i}(s)+\int_{s}^{t} e^{-(t-r) / \lambda_{i}} d L_{i}(r), \quad s<t, \quad i=1,2 .
$$

In this section we will discuss the methods to identify and filter out the spikes in the time series of spot prices. First of all, since our two factor framework is non-Markovian, this exercise is necessary to estimate the parameters for the two components of the model. Especially when spike behavior (frequency and/or size) appears in a non-stationary seasonal way (as for example seems to be the case for the Nord pool series), effective estimation of the model requires the separation of the non-stationary spikes path from the stationary path of the remaining daily variations. Further, besides serving estimation purposes, the identification of the individual factors is essential to apply the model to futures and other derivatives pricing (see Benth et al. [3]).

In the literature, most of the existing spot price models driven by processes including jumps are of jump diffusion type. When estimating a model of this type, the difficulty is to determine which price variations are caused by jumps and which ones are caused by the continuous part of the process. The easiest and most common way to deal with this problem is to fix a threshold (according to some criteria) above which price variations are considered to be caused by jumps.

For our purposes, these threshold methods are not appropriate. The reason is that they are designed to filter out the path of jumps whereas we need to identify the spike component $Y_{2}(t)$, i.e., we need to filter out the path of complete spikes (jumps followed by rapid mean reversion). 
One potential method to filter out the spikes is stochastic filtering. The aim of this procedure is to estimate the spike component at time $t$ given the price observations $X(s), s \leq t$, until time $t$ via the optimal filter

$$
E\left[f\left(Y_{2}(t)\right) \mid \mathcal{F}_{t}^{X}\right]
$$

where $\mathcal{F}_{t}^{X}$ denotes the filtration generated by the observations $X(t)$. In the case where both factors are Gaussian, the solution is explicitly given by the well known Kalman filter, which was used for calibrating Gaussian multifactor models of electricity prices in [2]. In our framework, since the spike component obviously has to be non-Gaussian, there is no explicit solution and Monte Carlo methods (particle filters) have to be employed in order to approximate the filter. However, when implementing the particle filter in our situation one encounters several difficulties, in particular:

- the filter is not easy to design when parameters are unknown;

- rare events such as spikes lead to sample impoverishment;

- sequential filtering makes less sense when the complete series is available for estimation.

Because of the above objections, we don't pursue the particle filter approach any further. The approach that we want to focus on in more detail in this section is to filter out spikes by adopting methods from nonparametric statistics. In this setting, the general idea is to consider the spike path as deterministic data given through a function $f(t)$ which is disturbed by Gaussian (autoregressive) noise given through the base component $Y_{1}(t)$ :

$$
X(t)=Y_{1}(t)+f(t) .
$$

We will assume the spikes path is of the form as proposed by our model, i.e. $f(t) \in \mathcal{O U}$ where $\mathcal{O U}$ is the space of all Ornstein-Uhlenbeck functions

$$
f(t)=\sum_{i=1}^{M} \alpha_{i} 1_{t \geq \tau_{i}} e^{-\left(t-\tau_{i}\right) / \lambda_{2}} .
$$

Here, $M \in \mathbb{N}$ denotes the number of spikes, $\alpha_{i} \in \mathbb{R}$ are the spike sizes, and $\tau_{i} \in \mathbb{R}$ are the starting times of the spikes in the spike path $f(t)$.

Let $N$ be the sample size of our price series and $X\left(t_{j}\right), j=1, \ldots, N$, the deseasonalized (log) price observations, equally spaced in time (one day). For notational convenience we introduce for a function $g(t)$ the notation $g(j):=$ $g\left(t_{j}\right)$. Then, according to our model,

$$
(X(j)-f(j))=Y_{1}(j), \quad j=1, \ldots, N,
$$

is a sequence of correlated Gaussian random variables. In order to pass to a sequence of iid Gaussian random variables suited for estimation purposes we can consider the transformation

$$
(\Delta X(j)-\Delta f(j)), \quad j=2, \ldots, N,
$$


where we define for a function $g(t)$

$$
\Delta g(j)=g(j)-e^{-1 / \lambda_{1}} g(j-1) .
$$

Then

$$
\Delta X(j)=Y_{1}(j)+f(j)-e^{-1 / \lambda_{1}}\left(Y_{1}(j-1)+f(j-1)\right)=\Delta Y_{1}(j)+\Delta f(j),
$$

and from the explicit solution of an Ornstein-Uhlenbeck process we get that $\Delta Y_{1}(j)$ is a sequence of iid Gaussian random variables. Taking (7) as a starting point, we propose in the following two methods to filter out the spikes path $f(t)$ from our data, i.e. to determine the spike number $M$ and spike sizes and times $\left(\alpha_{i}, \tau_{i}\right)$ : hard thresholding and the adapted Potts filter.

Hard thresholding Given the number $M$ of spikes and assuming the two mean reversion coefficients $\lambda_{1}$ and $\lambda_{2}$ to be known (estimated from the autocorrelation function), we know that the maximum likelihood estimator $\left(\alpha_{i}^{*}, \tau_{i}^{*}\right)$ of $\left(\alpha_{i}, \tau_{i}\right), \mathrm{i}=1, \ldots, \mathrm{M}$, is given by least squares estimation, i.e.

$$
\left(\alpha_{i}^{*}, \tau_{i}^{*}\right)=\arg \inf _{\left(\alpha_{i}, \tau_{i}\right)} \sum_{j=1}^{N}(\Delta X(j)-\Delta f(j))^{2} .
$$

However, the complexity of this optimization problem is of order $N^{M}$ which makes it unfeasible for implementation.

Instead, the hard thresholding method suggests to approximate the problem by placing the spikes one by one. To this end suppose we want to optimally place one spike given through

$$
f(t)=\alpha 1_{t \geq \tau} e^{-(t-\tau) / \lambda_{2}} .
$$

In this case, the maximum likelihood estimator

$$
\left(\alpha^{*}, \tau^{*}\right)=\arg \inf _{(\alpha, \tau)} \sum_{j=1}^{N}(\Delta X(j)-\Delta f(j))^{2}
$$

can be computed as

$$
\alpha^{*}=\frac{\sum \Delta X(j) \Delta f(j)}{\Delta f(j)^{2}}, \quad \tau^{*}=\arg \sup \frac{\left(\sum \Delta X(j) \Delta f(j)\right)^{2}}{\Delta f(j)^{2}} .
$$

It follows that the algorithm in order to place $M$ spikes optimally one by one can be formulated as follows:

1. Set $X^{(1)}=X$ and $X^{(m+1)}=X^{(m)}-f^{(m)}$ for $m=1, \ldots M$.

2. For every $m=1, \ldots M$, the $m^{\prime}$ th spike $f^{(m)}(t)=\alpha^{m} 1_{t \geq \tau^{m}} e^{-\left(t-\tau^{m}\right) / \lambda_{2}}$ is determined by

$$
\alpha^{m}=\frac{\sum \Delta X^{(m)}(j) \Delta f(j)}{\Delta f(j)^{2}}, \quad \tau^{m}=\arg \sup \frac{\left(\sum \Delta X^{(m)}(j) \Delta f(j)\right)^{2}}{\Delta f(j)^{2}} .
$$




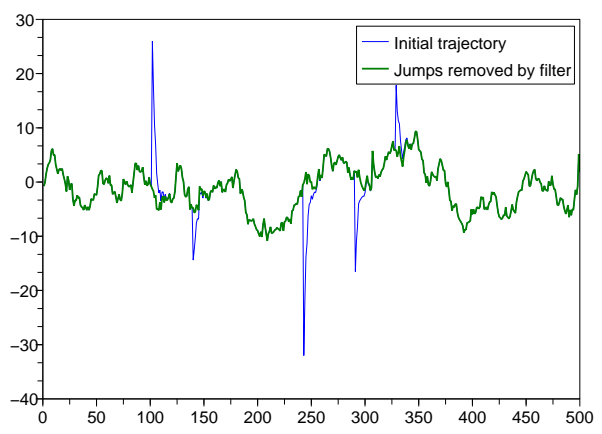

Figure 11: Performance of hard thresholding on simulated data.

In contrast to the global maximum likelihood estimation, the hard thresholding algorithm is of order $M N$ and thus well suited for implementation. The procedure stops, that is the optimal number $M$ of spikes is found, when the spike size becomes small according to some criteria (see the following section for an example of such a criteria).

In Figure 11 the hard thresholding method is tested on some simulated data, and in Figure 12 we applied hard thresholding to Powernext data. The procedure appears satisfactory, however, two remarks have to be made. Typically, the performance of the filter improves when a spike correlation length $\lambda_{2}$ is chosen that is shorter than the one obtained from the autocorrelation function. For Powernext data the autocorrelation function yields $\lambda_{2} \sim 2.5-4$, whereas $\lambda_{2} \sim 1-2$ is a better choice for the filter. This indicates that $\lambda_{2}$ captures some additional mean reversion not belonging to spike behavior when fitting the autocorrelation function. As for the first correlation length $\lambda_{1}$, the filter behaves in a rather robust way in the range $\lambda_{1}=10-100$. Secondly, we see that the hard thresholding algorithm works better for non-interacting spikes. When there is a cluster of spikes closely following each other, hard thresholding encounters problems in separating the spikes. In this situations, the adapted Potts filter presented in the following might be a better choice.

Adapted Potts filter In Winkler and Liebscher [19] the authors introduce a Potts filter which estimates a piecewise constant signal from noisy data by penalized least squares. The idea we propose in this paragraph is to adapt the Potts filter from [19] in order to detect spikes rather than piecewise constant signals disturbed by some noise. In the situation of spike detection the penalized 

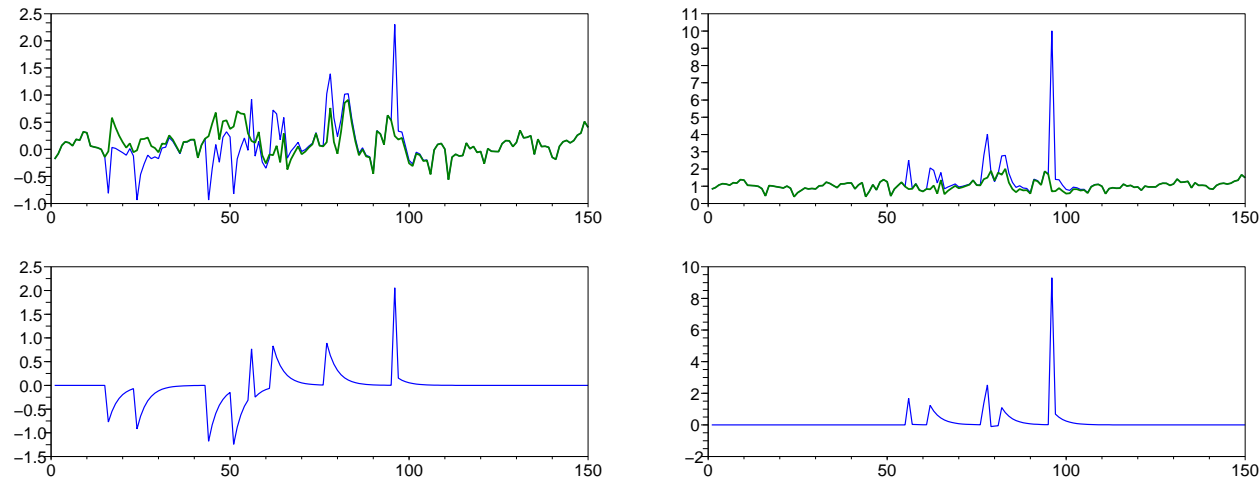

Figure 12: Performance on Powernext data. Left: in the log-price series both positive and negative spikes are present. Right: in the price series mostly positive spikes are present.

least squares optimization problem takes the following form:

$$
H\left(f^{*}\right)=\arg \inf _{f \in \mathcal{O} \mathcal{U}}\left(\gamma\left|\left\{t: f_{t^{-}} \neq f_{t}\right\}\right|+\sum_{j=1}^{N}(\Delta X(j)-\Delta f(j))^{2}\right) .
$$

Here $f_{t^{-}}$denotes the left limit of $f$ in $t$ (where we set $f_{0^{-}}=0$ ) and $\mid\left\{t: f_{t^{-}} \neq\right.$ $\left.f_{t}\right\} \mid$ is the number of spikes in the path of $f$. We thus want to find the spike path $f^{*}$ that is closest to our data in the least squares sense (after accounting for autoregressive noise) where, however, every spike is penalized by a weight $\gamma$.

In order to solve problem (8) we first consider the case of uncorrelated noise which corresponds to $\lambda_{1}=0$. In this situation problem (8) takes the form

$$
H\left(f^{*}\right)=\arg \inf _{f \in \mathcal{O} \mathcal{U}}\left(\gamma\left|\left\{t: f_{t^{-}} \neq f_{t}\right\}\right|+\sum_{j=1}^{N}(X(j)-f(j))^{2}\right),
$$

which can be explicitly solved by employing a dynamic programming argument. For this purpose we denote by

$$
B(r):=\arg \inf _{f \in \mathcal{O} \mathcal{U}}\left(\gamma\left|\left\{t \leq n: f_{t^{-}} \neq f_{t}\right\}\right|+\sum_{t \leq r}(X(j)-f(j))^{2}\right)
$$

the solution of the problem on the interval [1,r] (such that $B(N)=H\left(f^{*}\right)$ ). Further, we denote the cost of placing a spike on an interval $[r, s], 1 \leq r \leq s \leq N$, as

$$
H_{[r, s]}:=H_{[r, s]}(\mu):=\gamma+\sum_{j=r}^{s}\left(X(j)-\mu e^{-(j-r) / \lambda_{2}}\right)^{2},
$$


and respectively we denote as

$$
H_{[0, s]}=\sum_{j=1}^{s} X(j)^{2}
$$

the cost if now spike has been placed on $[1, s]$. Note here that $\mu$ does not denote the spike size (which above was denoted by $\alpha$ ) but the level $f(r)$ of the spike path at time $r$ (i.e. spike size $\alpha$ plus $f\left(r^{-}\right)$). Then, with the same arguments as in ([19]), we can represent $B(n), 1 \leq n \leq N$, recursively by employing dynamic programming:

$$
B(n)=\min _{0 \leq r \leq n}\left\{B(r-1)+\min _{\mu \in \mathbb{R}} H_{[r, n]}(\mu)\right\} .
$$

Here we set $B(-1):=0$ and $B(0):=0$. The algorithm to solve problem $(9)$ can then be formulated as follows:

1. For all $1 \leq r \leq s \leq N$ determine $\mu_{[r, s]}^{*}=\arg \min _{\mu} H_{[r, s]}(\mu)$ and $H_{[r, s]}\left(\mu_{[r, s]}^{*}\right)$. Determine further $H_{[0, s]}$ and set $\mu_{[0, s]}^{*}=0$ for all $1 \leq s \leq N$.

2. Determine $B(n)$ for all $1 \leq n \leq N$, keeping track of (at least one) $r_{n}^{*}$ giving the minimum in (10).

3. Determine recursively through $N, r_{N}^{*}, r_{r_{N}^{*}}^{*}, \ldots$ the spike number and spike times of the optimal path $f^{*}$. The optimal spike sizes are then implied by the corresponding $\mu_{\left[r_{N}^{*}, N\right]}^{*}, \mu_{\left[r_{r_{N}^{*}}^{*}, r_{N}^{*}\right]}^{*}, \ldots$

In the general case of autoregressive noise, i.e. $\lambda_{1} \in(0, \infty)$, problem (8) can no longer be solved explicitly with the above algorithm. In this situation the cost of placing a spike on an interval $[r, s], 1 \leq r \leq s \leq N$, takes the form

$$
\begin{aligned}
& H_{[r, s]}(\mu)=\gamma+\sum_{j=r}^{s}(\Delta X(j)-\Delta f(j))^{2} \\
& \quad=\left(\Delta X(r)-\left(\mu-e^{-1 / \lambda_{1}} f(r-1)\right)\right)^{2}+\sum_{j=r+1}^{s}\left(\Delta X(j)-\mu \Delta e^{-(j-r) / \lambda_{2}}\right)^{2} .
\end{aligned}
$$

We see that in order to solve for an optimal level $\mu$ on the interval $[r, s]$ we need to know the spike path level $f(r-1)$ at time $r-1$. In other words we can no longer place spikes optimally using local considerations only on the interval $[r, s]$, and therefore the dynamic programming argument is not applicable. Instead we propose to approximate the problem by substituting the optimal cost function $H_{[r, s]}\left(\mu_{[r, s]}^{*}\right)$ in 1 . of the above algorithm with

$$
K_{[r, s]}=\gamma+\sum_{j=r+1}^{s}\left(\Delta X(j)-X(r) \Delta e^{-(j-r) / \lambda_{2}}\right)^{2} .
$$


This amounts to assuming that the size of the optimally placed spike on the interval $[r, s]$ is equal to $\Delta X(r)$ (all the variation is explained by the spike) and then spike function at time $r$ is equal to $X(r)$. Obviously, the cost of placing spike of this type can then be computed locally. Also we set

$$
K_{[0, s]}=\sum_{j=1}^{s} X(j)^{2}
$$

for the cost of not placing a spike. As before we then introduce the recurrence relation

$$
B(n)=\min _{0 \leq r \leq n}\left\{B(r-1)+K_{[r, s]}\right\} .
$$

Note that in this case $B(N)$ is not the solution of problem (8) but of the corresponding problem where instead of optimizing over paths $f \in \mathcal{O U}$ we optimize over paths resulting from the type of spikes in (12). We use this optimization procedure in order to determine the spike number and times (but not spike sizes) by the following algorithm:

1. For all $1 \leq r \leq s \leq N$ determine $K_{[r, s]}$. Also determine $K_{[0, s]}$ for all $1 \leq s \leq N$

2. Determine $B(n)$ for all $1 \leq n \leq N$, keeping track of (at least one) $r_{n}^{*}$ giving the minimum in (4).

3. Determine recursively through $N, r_{N}^{*}, r_{r_{N}^{*}}^{*}, \ldots$ the spike number and spike times of the optimal path $f^{*}$.

Given the spike number $M$ and spike times $\tau_{1}, \ldots, \tau_{M}$ obtained from the above algorithm, the optimal spike sizes $\alpha_{1}^{*}, \ldots \alpha_{M}^{*}$ are then determined by global least squares optimization:

$$
\left(\alpha_{i}^{*}\right)=\arg \inf _{\alpha_{i}} \sum_{j=1}^{N}\left(\Delta X(j)-\Delta \sum_{i=1}^{M} \alpha_{i} 1_{j \geq \tau_{i}} e^{-\left(j-\tau_{i}\right) / \lambda_{2}}\right)^{2} .
$$

In Figure 13 the performance of the autoregressive Potts filter is compared to the hard thresholding method on Powernext data. We see that both filters deliver comparable and acceptable results.

\section{Case study: Modeling EEX spot prices}

We conclude this paper by estimating the two-factor Ornstein-Uhlenbeck model defined by (4)-(5) on the EEX time series. We choose to estimate our model on the spot price rather than on its logarithm to avoid introducing artificial downward jumps by the logarithmic transformation. We discuss both model specification and parameter estimation in four steps. 


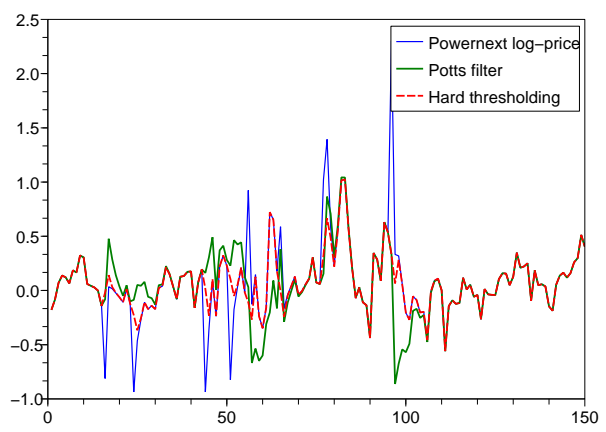

Figure 13: Comparison of the adapted Potts filter and the hard thresholding algorithm for spike detection.
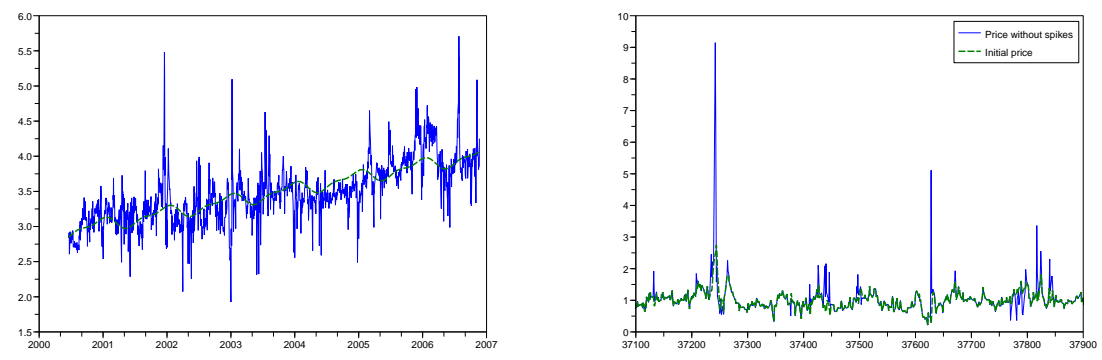

Figure 14: Left: Removing the seasonal component from the (logarithmic) EEX daily time series. The seasonal component is highest in winter (the year ticks on the graph correspond to January 1st). Right: spike detection using the hard thresholding procedure. The procedure is applied to the deseasonalized price series directly (rather than its logarithm), and only part of the data series is shown. 
Step 1 (Deseasonalizing the data): We fit the seasonality function (1) to logarithmic spot prices (multiplicative seasonality). This is carried out as described in Section 2. The deseasonalized spot price series is

$$
X_{t}^{\text {des }}=\frac{X_{t}}{e^{f(t)}} .
$$

The seasonal component of the EEX series is plotted in Figure 14, left graph.

Step 2 (Separation of components): The spike component in the deseasonalized spot price series can be identified by using one of the two methods described in the previous section (hard thresholding or Potts filter). Each of these methods has 3 tuning parameters: the correlation lengths $\lambda_{1}$ and $\lambda_{2}$, and a third parameter which determines how many spikes we want to remove.

Estimation from the autocorrelation function described in Section 2 gives $\lambda_{1}=99.4$ and $\lambda_{2}=2.9$ for the EEX series (this is different from the numbers given in Table 5 because the latter were computed for the logarithm of the spot price). As explained in Section 4, in practice, the hard thresholding algorithm is insensitive to the value of $\lambda_{1}$ in the range of $10-100$ and relatively sensitive to the value of $\lambda_{2}$, so that best results (the smallest residual volatility for the same number of spikes removed) are obtained with $\lambda_{2}$ between 1 and 2 .

The number of spikes to remove can be determined by fixing some "target noise level" and then removing spikes with the hard thresholding method, or decreasing the penalty parameter $\gamma$ in the Potts filter until the standard deviation of returns of the residual signal (the signal from which spikes have been removed) reaches this target noise level. The target noise level represents our a priori knowledge of the noise level of the base signal. One possible way to do assess this quantity is to remove some percentage $\epsilon$ of returns with highest absolute value, and compute the standard deviation of the remaining returns. The idea is that $\epsilon$ biggest returns correspond to spikes. This procedure is not sensitive to outliers (occasional very big returns).

For the actual estimation, we used the hard thresholding method taking $\lambda_{1}=100, \lambda_{2}=1$. To compute the target noise level, the percentage of returns to be removed was set to $\epsilon=5 \%$. This gives the target standard deviation $\sigma^{*}=0.154$ (the standard deviation computed using all available returns is 0.38 ). With this criterion, the adapted Potts filter algorithm detected 115 spikes in a series of 6.5 years with the penalty parameter $\gamma=0.13$. The hard thresholding algorithm on the other hand needed to place only 62 jumps to achieve the prescribed standard deviation. Despite this, the residual signal for the Potts filter had a higher excess kurtosis than that for the hard thresholding algorithm, suggesting that in the former case some spikes/jump persist after the filtering procedure. We therefore concentrate on the hard thresholding algorithm for the rest of this study. Figure 14, right graph, illustrates the spike detection using the hard thresholding procedure.

Step 3 (Distribution of the base signal): In the case of EEX series, the increments of the base signal have a skewness of -0.008 (down from 2.62 for the initial deseasonalized series including spikes) and an excess kurtosis of 1.05 
(down from 113). We conclude that the base signal is sufficiently close to Gaussian to be modeled by an $\mathrm{AR}(1)$ process with Gaussian increments. The parameters of this process can be estimated by maximum likelihood, which gives $e^{-1 / \lambda_{1}}=0.85 \Rightarrow \lambda_{1}=6.2$ and $\sigma=0.148$. The difference between this value and the value of $\lambda_{1}$ estimated from the autocorrelation function may be explained by the presence of not two but three exponential components in the autocorrelation function. Indeed, fitting the autocorrelation function with three exponentials, we obtain $\lambda_{2}=1.69, \lambda_{1}=14.4$ plus a third factor with a very long decay rate. However, for the sake of simplicity, we concentrate on the two-factor model.

Step 4 (Distribution of the spike signal): We need to estimate the spike intensity and the distribution of the spike amplitude. Since we don't observe any seasonal behavior in spike occurrence on EEX data we assume a constant spike intensity denoted by $\lambda$. Then $\lambda$ is determined as

$$
\lambda=\frac{\text { number of spike detected }}{\text { total number of data points }}
$$

which gives $\lambda=0.037$ in the case of the EEX series. To model the spike size distribution, we choose the Pareto law with distribution function

$$
P(Z>z)=\left(\frac{z}{z_{0}}\right)^{-\alpha} .
$$

There are several reasons for this choice:

- Since the spike amplitudes are from bounded below by a threshold, we need a distribution supported on the interval $\left[z_{0}, \infty\right)$.

- The number of data points for estimation is limited (62 spikes in the case of the EEX series) therefore we cannot estimate a distribution with many parameters.

- Figure 15 shows that the empirical CDF of the spike amplitude distribution is very close to a straight line on a log-log scale, which is an indication of a power-law decay.

For the EEX series, the threshold $z_{0}$ was taken equal to 0.45 (smallest spike amplitude), and the decay rate $\alpha$ is equal to 1.44 (this was found by fitting a straight line to the empirical CDF graph in the log-log scale).

In summary, we have the following model for the EEX price:

$$
\begin{aligned}
& X(t)=e^{f(t)}\left(Y_{1}(t)+Y_{2}(t)\right) \\
& d Y_{1}(t)=-\lambda_{1}^{-1} Y_{1}(t)+\sigma d W(t) \\
& d Y_{2}(t)=-\lambda_{2}^{-1} Y_{2}(t)+d L(t),
\end{aligned}
$$

where $W$ is Brownian motion and $L$ is a compound Poisson process with intensity $\lambda$ and with jump size following Pareto distribution with parameters $z_{0}$ and 


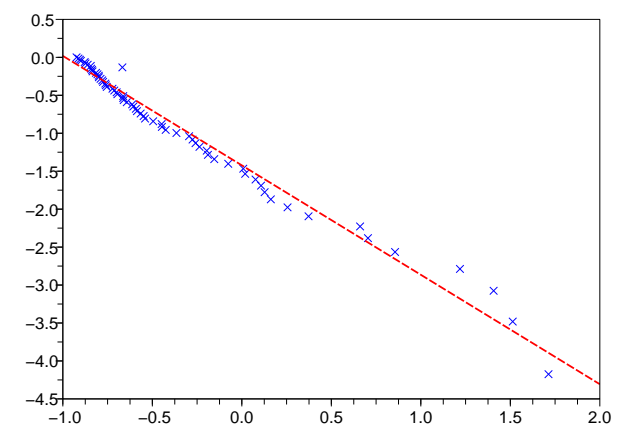

Figure 15: Empirical CDF of spike size in log-scale.

$\alpha$. This model specification can be used to simulate price trajectories. Figure 16 confirms that indeed the model mimics the path behavior of the spot price series very well.

\section{References}

[1] M. T. BARLow, A diffusion model for electricity prices, Mathematical Finance, 12 (2002), pp. 287-298.

[2] M. T. Barlow, Y. Gusev, and L. Manpo, Calibration of multifactor models in electricity markets, International Journal of Theoretical and Applied Finance, 7 (2004), pp. 101-120.

[3] F. E. Benth, J. Kallsen, and T. Meyer-Brandis, A non-Gaussian Ornstein-Uhlenbeck process for electricity spot price modeling and derivatives pricing, Applied Mathematical Finance, 14 (2007), pp. 153-169.

[4] L. De HaAn, H. Drees, and S. Resnick, How to make a Hill plot, Annals of Statistics, (2000).

[5] S. Deng, Stochastic models of energy commodity prices and their applications: mean-reversion with jumps and spikes, October 1999. Working paper, Georgia Institute of Technology.

[6] S.-J. Deng And W. Jiang, Lévy process-driven mean-reverting electricity price model: the marginal distribution analysis, Decision Support Systems, 40 (2005), pp. 483-494. 

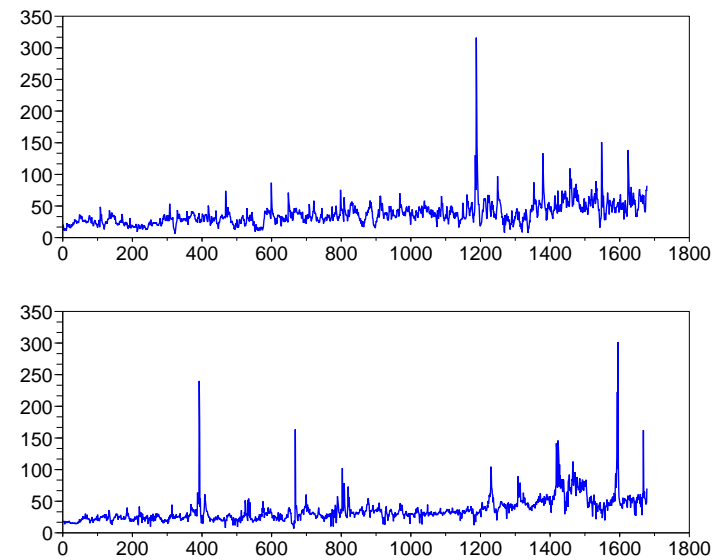

Figure 16: Comparison of the real EEX series and the simulated series with estimated parameters. Which is which? (The market series is the lower one).

[7] A. Escribano, J. I. Pena, and P. Villaplana, Modelling electricity prices: International evidence. Universidad Carlos III de Madrid working paper, 2002.

[8] K. R. French, Stock returns and the weekend effect, Journal of Financial Economics, 8 (1980), pp. 55-69.

[9] H. Geman, Commodities and Commodity Derivatives, Wiley Finance, 2005.

[10] H. Geman And A. Roncononi, Understanding the fine structure of electricity prices, Journal of Business, (2006).

[11] T. Kanamura and K. Ohashi, A structural model for electricity prices with spikes: Measurement of jump risk and optimal policies for hydropower plant operation. Working paper, Hitotsubashi University.

[12] C. Knittel And M. Roberts, An empirical examination of deregulated electricity prices. POWER WP-087, University of California Energy Institute, 2001.

[13] A. Leon And A. Rubia, Testing for weekly seasonal unit roots in the spanish power pool, in In Modelling Prices in Competitive Electricity Markets., D. W. Bunn, ed., Wiley Series in Financial Economics, Wiley, 2004, pp. $177-189$.

[14] A. Misionek, S. Trueck, And R. Weron, Point and interval forecasting of spot electricity prices: Linear vs. non-linear time series models., Studies in Nonlinear Dynamics \& Econometrics, 10 (2006). 
[15] Platts, Platts methodology and specifications guide: North american electricity. Available from www.platts.com.

[16] G. W. Schwert, Anomalies and market efficiency, in Handbook of the Economics of Finance, 2002, pp. 937-972.

[17] P. Villaplana, Pricing power derivatives: A two-factor jump-diffusion approach. EFMA 2004 Basel Meetings Paper. Available at SSRN: http://ssrn.com/abstract=493943, September 2003.

[18] R. Weron, Heavy tails and electricity prices. Research Report HSC/05/2, Hugo Steinhaus Center for Stochastic Methods, 2005.

[19] G. Winkler AND V. LiEBscher, Smoothers for discontinuous signals, Nonparametric Statistics, 14 (2002), pp. 203-222. 